\title{
Two-dimensional magnetic semiconductors with room Curie temperatures
}

\author{
Jing-Yang You $\odot,{ }^{1}$ Zhen Zhang, ${ }^{1}$ Xue-Juan Dong, ${ }^{1}$ Bo Gu $\odot,{ }^{2,3, *}$ and Gang Su $\odot^{1,2,3, \dagger}$ \\ ${ }^{1}$ School of Physical Sciences, University of Chinese Academy of Sciences, Beijing 100049, China \\ ${ }^{2}$ Kavli Institute for Theoretical Sciences and CAS Center for Excellence in Topological Quantum Computation, \\ University of Chinese Academy of Sciences, Beijng 100190, China \\ ${ }^{3}$ Physical Science Laboratory, Huairou National Comprehensive Science Center, 101400 Beijing, China
}

(Received 29 August 2019; published 2 January 2020)

\begin{abstract}
We propose two-dimensional (2D) Ising-type ferromagnetic semiconductors $\mathrm{TcSiTe}_{3}, \mathrm{TcGeSe}_{3}$, and $\mathrm{TcGeTe}_{3}$ with high Curie temperatures around 200-500 K. Owing to large spin-orbit couplings, the large magnetocrystalline anisotropy energy (MAE), large anomalous Hall conductivity, and large magneto-optical Kerr effect were discovered in these intriguing $2 \mathrm{D}$ materials. By comparing all possible $2 \mathrm{D} M \mathrm{GeTe}_{3}$ materials $(M=3 d, 4 d, 5 d$ transition metals), we found a large orbital moment around $0.5 \mu_{B}$ per atom and a large MAE for $\mathrm{TcGeTe}_{3}$. The large orbital moments are revealed to be from the comparable crystal fields and electron correlations in these Tc-based 2D materials. The microscopic mechanism of the high Curie temperature is also addressed. Our findings reveal the unique magnetic behaviors of 2D Tc-based materials and present a family of 2D ferromagnetic semiconductors with large MAE and Kerr rotation angles that would have wide applications in designing spintronic devices.
\end{abstract}

DOI: 10.1103/PhysRevResearch.2.013002

\section{INTRODUCTION}

Spin-orbit coupling (SOC) describes the relativistic interaction between the spin and orbital momentum of electrons [1]. Spin-orbit coupling can drive rich phenomena, such as magnetic anisotropy [2], spin relaxation [3], magnetic damping [4], anisotropic magnetoresistance [5], and the anomalous Hall effect [6]. Recently, the term spin orbitronics was proposed to cover the expanding research field, where SOC is a key concept $[1,7,8]$. Combining strong SOC and magnetism, many intriguing physical phenomena can be achieved, including current-driven magnetization reversal [9-11], domain-wall propagation [12,13], and currentdriven skyrmion motion [14-16]. Transition metals are usually candidates to realize these phenomena and play important roles in spin orbitronics.

Magnetic anisotropy is one of the fundamental properties of magnetic materials. It is a key issue in recent advances in two-dimensional (2D) magnetic semiconductors [17-20]. According to the Mermin-Wagner theorem [21], at finite temperatures, the quantum spin- $S$ Heisenberg model with isotropic and finite-range exchange interactions in 1D or 2D lattices can be neither ferromagnetism nor antiferromagnetism. Thus, to stabilize long-range ferromagnetic order in 2D magnetic semiconductors at finite temperature, a large

\footnotetext{
*gubo@ucas.ac.cn

†gsu@ucas.ac.cn

Published by the American Physical Society under the terms of the Creative Commons Attribution 4.0 International license. Further distribution of this work must maintain attribution to the author(s) and the published article's title, journal citation, and DOI.
}

magnetic anisotropy, which takes the systems away from the isotropic Heisenberg model, is extremely important.

The magneto-optical Kerr effect (MOKE), which is closely related to SOC, is a basic magneto-optic effect. It describes the action whereby the plane-polarized light reflected from a magnetized material becomes elliptically polarized and the plane of polarization is rotated. The MOKE is widely used to probe the electronic structure of magnetic materials. Many exciting phenomena related to the MOKE have been discovered, such as quantum confinement effects [22], oscillations of the Kerr rotation with magnetic layer thickness [23], and strong correlations between the MOKE and magnetic anisotropies [24]. Due to the application of the MOKE to the readout process in magneto-optical storage devices, much effort has been devoted to searching for materials with large Kerr rotation angles.

In this paper we propose three stable 2D ferromagnetic semiconductors $\mathrm{TcSiTe}_{3}, \mathrm{TcGeSe}_{3}$, and $\mathrm{TcGeTe}_{3}$, which share the same crystal structure as the recently discovered 2D magnetic semiconductor $\mathrm{CrGeTe}_{3}$ [19]. These Tc-based 2D materials have not been observed experimentally yet. The Monte Carlo simulations give Curie temperatures of 538, 212 , and $187 \mathrm{~K}$ for $\mathrm{TcSiTe}_{3}, \mathrm{TcGeSe}_{3}$, and $\mathrm{TcGeTe}_{3}$ monolayers, respectively, which are much higher than the Curie temperature in $\mathrm{CrGeTe}_{3}$. The calculations show that these Tc-based materials have a spin moment of about $2 \mu_{B}$ and an extraordinarily large orbital moment of about $0.5 \mu_{B}$ per $\mathrm{Tc}$ atom. The large orbital moment comes from the partially occupied $d$ orbitals, and the partial occupation of $d$ orbitals is due to the comparable crystal fields and electron correlations in these Tc-based 2D materials. As a result, a large SOC is obtained in these materials. Due to the large SOC, a large magnetocrystalline anisotropy energy (MAE) is formed, indicating the Ising behavior of these $2 \mathrm{D}$ materials with 
out-of-plane magnetization. In addition, a large Kerr rotation angle of about $3.6^{\circ}$ is achieved in these Tc-based materials, which is much larger than the value of $0.8^{\circ}$ in metal Fe. Large anomalous Hall conductivity of about $7.5 \times 10^{2}(\Omega \mathrm{cm})^{-1}$ in $p$-type $\mathrm{TcGeTe}_{3}$ and $1.1 \times 10^{3}(\Omega \mathrm{cm})^{-1}$ in $n$-type $\mathrm{TcGeTe}_{3}$ is comparable to the anomalous Hall conductivity of $7.5 \times$ $10^{2}(\Omega \mathrm{cm})^{-1}$ in bulk Fe $[25,26]$ and $4.8 \times 10^{2}(\Omega \mathrm{cm})^{-1}$ in bulk Ni [27]. The microscopic mechanism of high Curie temperature in these Tc-based materials is also discussed.

\section{COMPUTATIONAL METHOD}

Our first-principles calculations were based on the densityfunctional theory as implemented in the Vienna ab initio simulation package (VASP) [28], using the projector augmented wave method [29]. The generalized gradient approximation (GGA) with Perdew-Burke-Ernzerhof [30] realization was adopted for the exchange-correlation functional. We set the on-site Hubbard interaction $U=2.3 \mathrm{eV}$ and Hund coupling $J=0.3 \mathrm{eV}$ [31] in considering electron correlation of $4 d$ electrons of Tc atoms, and the effective $U_{\text {eff }}=U-J=2 \mathrm{eV}$, because the reasonable $U_{\text {eff }}$ is about $2 \mathrm{eV}$ for $4 d$ electrons. The plane-wave cutoff energy was set to $550 \mathrm{eV}$. A MonkhorstPack $k$-point mesh [32] of size $13 \times 13 \times 1$ was used for the Brillouin zone (BZ) sampling. The structure relaxation considering both the atomic positions and lattice vectors was performed by the conjugate gradient scheme until the maximum force on each atom was less than $0.0001 \mathrm{eV} / \AA$, and the total energy was converged to $10^{-8} \mathrm{eV}$ with the Gaussian smearing method. To avoid unnecessary interactions between the monolayer and its periodic images, the vacuum layer was set to $15 \AA$. The phonon frequencies were calculated using a finite-displacement approach as implemented in the PHONOPY code [33], in which a $3 \times 3 \times 1$ supercell and a displacement of $0.01 \AA$ from the equilibrium atomic positions were employed. The WANNIER90 code [34] was used to construct an effective tight-binding Hamiltonian and to calculate the optic conductivity and the anomalous Hall conductivity.

\section{RESULTS}

The crystal structure of $\mathrm{TcSiTe}_{3}, \mathrm{TcGeSe}_{3}$, and $\mathrm{TcGeTe}_{3}$ monolayers from the prototypal $\mathrm{CrGeTe}_{3}$ monolayer is depicted in Fig. 1(a), where the space group is $P \overline{3} 1 m$ (No. 191). To determine the ground state of $\mathrm{TcSiTe}_{3}, \mathrm{TcGeSe}_{3}$, and $\mathrm{TcGeTe}_{3}$ monolayers, in the absence of SOC, we calculated the total energy for ferromagnetic (FM) and antiferromagnetic (AFM) configurations as a function of lattice constant and found that the FM state has an energy lower than the AFM state. The optimized lattice constants of $2 \mathrm{D} \mathrm{TcSiTe}_{3}$, $\mathrm{TcGeSe}_{3}$, and $\mathrm{TcGeTe}_{3}$ were calculated as 6.821, 6.379, and $7.029 \AA$, respectively, which are reasonable according to the radius of atoms.

To confirm the stability of these three monolayers, their phonon spectra have been calculated. There is no imaginary frequency mode in the whole Brillouin zone, as shown in Figs. S1(a)- S1(c) in the Supplemental Material [35], indicating that they are kinetically stable. We have checked the stability of these structures with different $U_{\text {eff }}(1$ and $3 \mathrm{eV})$, and the results show that these structures are always stable. To (a)

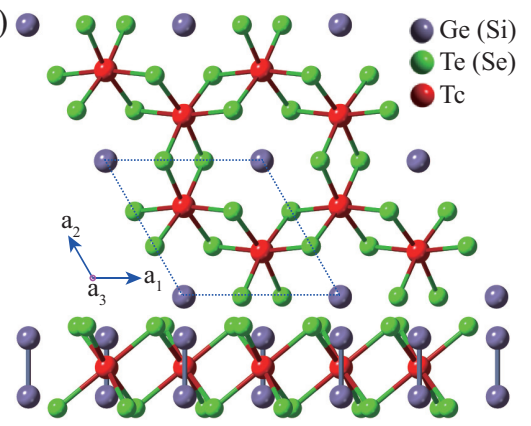

(b)

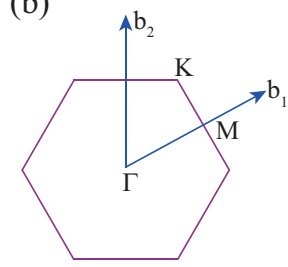

FIG. 1. (a) Top and side views of the crystal structure of $\mathrm{TcSiTe}_{3}$, $\mathrm{TcGeSe}_{3}$, and $\mathrm{TcGeTe}_{3}$ monolayers. The primitive cell is delineated by a dotted line box. The Tc atoms form a honeycomb lattice. (b) First Brillouin zone.

further examine the thermal stability, we performed $a b$ initio molecular dynamics simulations using a $4 \times 4 \times 1$ supercell containing 160 atoms. After being heated at 300 and $500 \mathrm{~K}$ for 6 ps with a time step of $3 \mathrm{fs}$, only little structural and energetic changes occur, as shown in Figs. S2(d)- S2(f) in [35], implying that $\mathrm{TcSiTe}_{3}, \mathrm{TcGeSe}_{3}$, and $\mathrm{TcGeTe}_{3}$ monolayers are dynamically stable.

The structural stabilities of $\mathrm{TcSiTe}_{3}, \mathrm{TcGeSe}_{3}$, and $\mathrm{TcGeTe}_{3}$ are also examined by the formation energy, which is calculated by $E_{f}=E\left(\mathrm{Tc} A B_{3}\right)-E(\mathrm{Tc})-E(A)-3 E(B)$, where $E(\mathrm{Tc}), E(A)$, and $E(B)$ are the total energies of the bulk $\mathrm{Tc}, \mathrm{Ge}(\mathrm{Si})$, and $\mathrm{Se}(\mathrm{Te})$ crystals, respectively. The negative values obtained are $E_{f}=-0.856,-1.582$, and $-0.694 \mathrm{eV}$ for $\mathrm{TcSiTe}_{3}, \mathrm{TcGeSe}_{3}$, and $\mathrm{TcGeTe}_{3}$, respectively. For the $\mathrm{CrGeTe}_{3}$ monolayer, which was discovered in a recent experiment [19], the formation energy was calculated as $-1.140 \mathrm{eV}$ by the same method. The comparable formation energy of $\mathrm{TcSiTe}_{3}, \mathrm{TcGeSe}_{3}$, and $\mathrm{TcGeTe}_{3}$ with $\mathrm{CrGeTe}_{3}$ suggests that these Tc-based materials may also be feasible in experiment.

Since $\mathrm{TcSiTe}_{3}, \mathrm{TcGeSe}_{3}$, and $\mathrm{TcGeTe}_{3}$ have similar properties, we will take $\mathrm{TcSiTe}_{3}$ in the following analysis, and the calculated results of $\mathrm{TcGeSe}_{3}$ and $\mathrm{TcGeTe}_{3}$ are shown in the Supplemental Material [35]. The partial density of states (PDOS) of the $\mathrm{TcSiTe}_{3}$ monolayer was calculated by the GGA $+U$ method, as illustrated in Fig. 2(a). Because of the octahedral crystal field for Tc atoms, the $d$ orbitals of the Tc atoms are split into threefold $t_{2 g}$ orbitals and twofold $e_{g}$ orbitals. For $\mathrm{Tc}^{2+}\left(4 d^{5}\right)$ in the $\mathrm{TcSiTe}_{3}$ monolayer, the spin moment $S=2 \mu_{B}$ and orbital moment $L=0.6 \mu_{B}$ are obtained. The results can be understood by the following electron configurations: 3 spin-up electrons and 0.6 spin-down electrons occupy $t_{2 g}$ orbitals, and 0.5 spin-up electrons and 0.9 spin-down electrons occupy $e_{g}$ orbitals. This reflects that the crystal field and Coulomb interaction $U$ are comparable for $4 d$ electrons of Tc. In contrast, for $\mathrm{Cr}^{2+}\left(3 d^{4}\right)$ in the $\mathrm{CrGeTe}_{3}$ monolayer ( $U$ is taken as $4 \mathrm{eV}$ ), 3 spin-up electrons are in $t_{2 g}$ orbitals and 1 spin-up electron is in $e_{g}$ orbitals, which gives rise to the spin moment $S=4 \mu_{B}$ and orbital moment $L=0$, as shown in Fig. 2(b). This means that the Coulomb interaction $U$ is much larger than the crystal field for $3 d$ electrons of $\mathrm{Cr}$. These results can also be obtained by integrating the total density of states below the Fermi level for spin-up and spin-down electrons for $t_{2 g}$ and $e_{g}$ orbitals, 

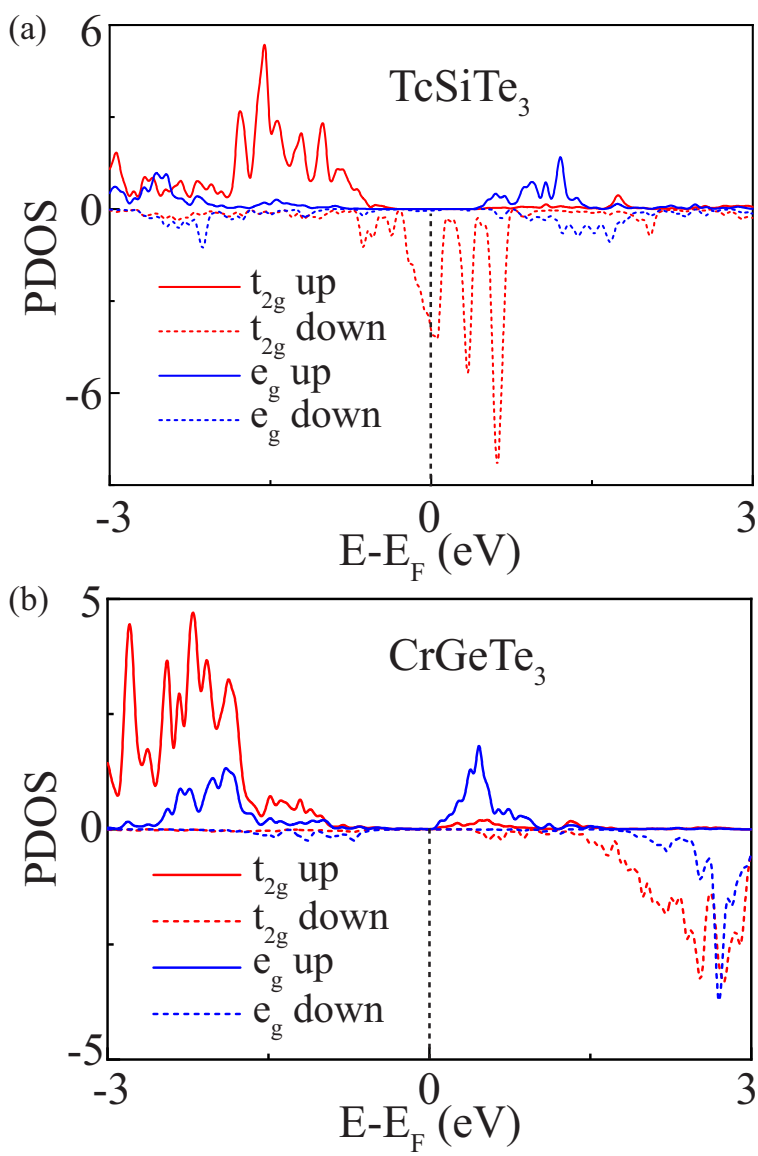

FIG. 2. Partial density of states of (a) $\mathrm{TcSiTe}_{3}$ and (b) $\mathrm{CrGeTe}_{3}$ monolayers, calculated by the GGA $+U$ method.

respectively. The SOC is calculated by $H_{\mathrm{SOC}}=\lambda \mathbf{S} \cdot \mathbf{L}$, where $\lambda$ is the coefficient of SOC and $\mathbf{S}$ and $\mathbf{L}$ represent the spin and orbital moment operators, respectively. Because of the large $\lambda$, which is related to the atomic number and large orbital moment $L$, a much larger SOC is expected in the $\mathrm{TcSiTe}_{3}$ monolayer than that in the $\mathrm{CrGeTe}_{3}$ monolayer.

The electronic band structure of the $\mathrm{TcSiTe}_{3}$ monolayer was calculated by the GGA $+U$ method, as shown in Fig. 3(a). It is a Weyl half-metal, where only one species of electron spin appears at the Fermi level. At the high-symmetry $\Gamma-K$ and $\Gamma-M$ lines, Weyl nodes exist. Considering the inversion and $C_{3 v}$ symmetries, there are 12 Weyl points in total
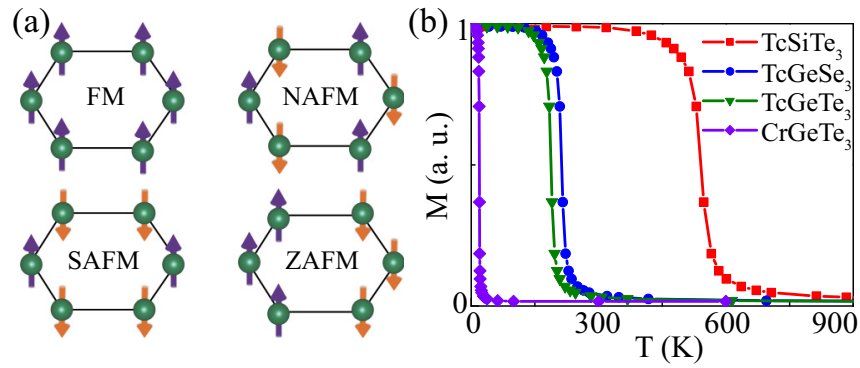

FIG. 4. (a) Possible spin configurations of Tc atoms on honeycomb lattice: FM, Néel AFM, stripe AFM, and zigzag AFM. (b) Temperature dependence of the normalized magnetic moment of $\mathrm{TcSiTe}_{3}, \mathrm{TcGeSe}_{3}, \mathrm{TcGeTe}_{3}$, and $\mathrm{CrGeTe}_{3}$ monolayers by Monte Carlo simulations.

within the BZ. To demonstrate the effect of SOC, the electronic band structure was calculated by the GGA $+\mathrm{SOC}+U$ method, as plotted in Fig. 3(b). As a result of including SOC, the band gap was opened for the $\mathrm{TcSiTe}_{3}$ monolayer. To correctly estimate the band gap, since the GGA-type calculations usually underestimate the band gap, the HeydScuseria-Ernzerhof hybrid functional method HSE06 was also employed. The HSE06 calculation shows that the band gap of the $\mathrm{TcSiTe}_{3}$ monolayer becomes $0.4 \mathrm{eV}$, as shown in Fig. 3(c). Details of electronic band structures are given in [35].

Due to the large SOC, there is a high expectation of a large magnetic anisotropy for $\mathrm{TcSiTe}_{3}, \mathrm{TcGeSe}_{3}$, and $\mathrm{TcGeTe}_{3}$ monolayers. To study magnetic anisotropy in these monolayers, we calculated the total energy with possible spin configurations of Tc atoms on a honeycomb lattice, including paramagnetic (PM), FM, Néel antiferromagnetic (NAFM), stripe AFM (SAFM), and zigzag AFM (ZAFM) configurations, as shown in Fig. 4(a). The $\mathrm{CrGeTe}_{3}$ monolayer was also calculated in the same way for comparison. The results are summarized in Table I. One can observe that the out-of-plane FM $\left(\mathrm{FM}^{z}\right)$ state has the lowest energy among the possible spin configurations. The magnetic anisotropy between the in-plane magnetic configurations $\mathrm{FM}^{x}$ and $\mathrm{FM}^{y}$ and the out-of-plane magnetic configurations $\mathrm{FM}^{z}$ in Tc-based materials is extraordinarily larger than that in $\mathrm{CrGeTe}_{3}$, as noted in Table I. We further calculated the energies for FM configurations by rotating the magnetic direction deviated from the $z$ axis and found that the $\mathrm{FM}^{z}$ state is the most energetically favorable, which shows an Ising behavior of the
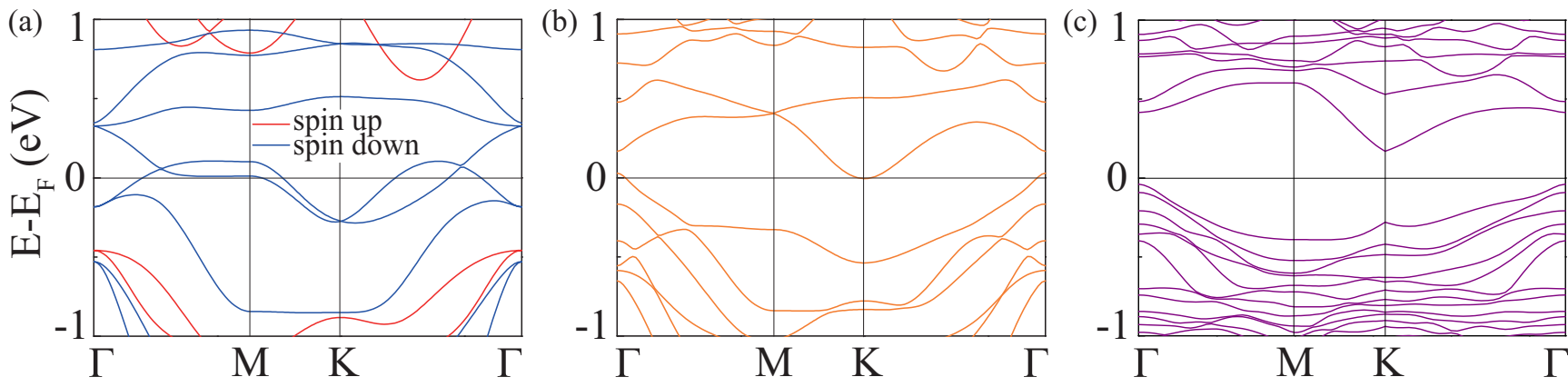

FIG. 3. Electronic band structures of $\mathrm{TcSiTe}_{3}$ calculated by the (a) GGA $+U$, (b) GGA $+\mathrm{SOC}+U$, and (c) HSE06 methods. 
TABLE I. Total energy $E_{\text {tot }}$ per unit cell for $\mathrm{TcSiTe}_{3}, \mathrm{TcGeSe}_{3}$, and $\mathrm{TcGeTe}_{3}$ monolayers (in meV, relative to $E_{\text {tot }}$ of the $\mathrm{FM}^{z}$ ground state) for several spin configurations of Tc atoms (see Fig. 4), calculated by the GGA+SOC $+U$ method. The spin moment $\langle S\rangle$ and orbital moment $\langle O\rangle$ (in units of $\mu_{B}$ ), single ion anisotropy (in meV) between the out-of-plane and in-plane FM configurations, exchange interaction $J$ (in meV), and Curie temperature $T_{\text {Curie }}$ (in $\mathrm{K}$ ) are calculated. The $\mathrm{CrGeTe}_{3}$ monolayer is also calculated with the experimental lattice constant [19] for comparison.

\begin{tabular}{lcrrrrrrrrrrr}
\hline \hline Monolayer & FM $^{z}$ & \multicolumn{1}{c}{ FM $^{x}$} & \multicolumn{1}{c}{ FM $^{y}$} & NAFM $^{z}$ & SAFM $^{z}$ & \multirow{2}{*}{ ZAFM $^{z}$} & PM & $\langle S\rangle$ & $\langle O\rangle$ & SIA & $J$ & $T_{\text {Curie }}(\mathrm{K})$ \\
\hline $\mathrm{TcSiTe}_{3}$ & 0.0 & 52.1 & 25.9 & 310.4 & 165.1 & 122.0 & 2000 & 1.866 & 0.540 & -42.5 & 7.625 & 538 \\
$\mathrm{TcGeSe}_{3}$ & 0.0 & 53.6 & 211.9 & 295.3 & 95.9 & 138.0 & 2065 & 1.884 & 0.515 & -37.7 & 2.997 & 212 \\
$\mathrm{TcGeTe}_{3}$ & 0.0 & 112.1 & 289.7 & 277.0 & 84.7 & 114.2 & 2300 & 1.991 & 0.562 & -26.5 & 2.647 & 187 \\
$\mathrm{CrGeTe}_{3}$ & 0.0 & 3.7 & 3.8 & 143.6 & 84.7 & 4.3 & 6000 & 3.614 & 0.004 & 0.032 & 0.066 & 19 \\
\hline \hline
\end{tabular}

$\mathrm{TcSiTe}_{3}, \mathrm{TcGeSe}_{3}$, and $\mathrm{TcGeTe}_{3}$ monolayers. The calculation reveals that the magnetic anisotropy originates from the single-ion anisotropy (SIA), which can be calculated by four ordered spin states [36]. The results in Table I show that, for $\mathrm{TcSiTe}_{3}, \mathrm{TcGeSe}_{3}$, and TcGeTe $\mathrm{Te}_{3}$ monolayers, SIA is found to be negative and large, which determines a strong Ising-type behavior with out-of-plane magnetization. For the $\mathrm{CrGeTe}_{3}$ monolayer, SIA is negligible and approaches zero, which indicates the Heisenberg-like behavior with weak magnetic anisotropy.

Thus, the magnetism in $\mathrm{TcSiTe}_{3}, \mathrm{TcGeSe}_{3}$, and $\mathrm{TcGeTe}_{3}$ monolayers can be described by the Ising-type Hamiltonian $H_{\text {spin }}=-\sum_{\langle i, j\rangle} J S_{i}^{z} S_{j}^{z}$, where $J$ represents the nearestneighbor exchange integral, $S_{i, j}^{z}$ is the $z$ component of the spin operator, and $\langle i, j\rangle$ denotes the summation over the nearest neighbors. Further, $J$ can be determined by the difference of energies between the $\mathrm{FM}^{z}$ configuration and the AFM configuration, which possesses the lowest energy among those AFM configurations. In our cases, the $\mathrm{ZAFM}^{z}$ configuration has the lowest energy for $\mathrm{TcSiTe}_{3}$ and $\mathrm{CrGeTe}_{3}$ monolayers, and the $\mathrm{SAFM}^{z}$ configuration has the lowest energy for $\mathrm{TcGeSe}_{3}$ and $\mathrm{TcGeTe}_{3}$ monolayers, as shown in Table I. As a result, $J$ was estimated to be 7.625, 2.997, 2.647, and $0.066 \mathrm{meV}$ for $\mathrm{TcSiTe}_{3}, \mathrm{TcGeSe}_{3}, \mathrm{TcGeTe}_{3}$, and $\mathrm{CrGeTe}_{3}$, respectively.

Based on the above Ising Hamiltonian and the estimated exchange parameter $J$, the Monte Carlo (MC) simulation was carried out to calculate the Curie temperatures of these $2 \mathrm{D}$ materials [37]. The MC simulation was performed on a $60 \times 60$ $2 \mathrm{D}$ honeycomb lattice using $10^{6}$ steps for each temperature. The magnetic moment as a function of temperature is shown in Fig. 4(b). It can be seen that the normalized magnetic moment decreases rapidly to vanish at Curie temperature of about 538, 212, 187, and $19 \mathrm{~K}$ for $\mathrm{TcSiTe}_{3}, \mathrm{TcGeSe}_{3}, \mathrm{TcGeTe}_{3}$, and $\mathrm{CrGeTe}_{3}$ monolayers, respectively. The results indicate that $\mathrm{TcSiTe}_{3}, \mathrm{TcGeSe}_{3}$, and $\mathrm{TcGeTe}_{3}$ monolayers can be potential candidates for high-temperature $2 \mathrm{D}$ ferromagnetic semiconductors.

Due to the large SOC in these ferromagnetic semiconductors, a large anomalous Hall conductivity (AHC) is expected. We calculated the intrinsic AHC due to the Berry curvature of the electronic band structure as shown in Fig. 5(a). The magnitude of AHC $\sigma_{x y}$ for the $p$-type $\mathrm{TcGeTe}_{3}$ can reach $7.5 \times 10^{2}(\Omega \mathrm{cm})^{-1}$ and for the $n$-type $\mathrm{TcGeTe}_{3}$ it can be up to $1.1 \times 10^{3}(\Omega \mathrm{cm})^{-1}$. That for the $p$ - or $n$-type $\mathrm{TcSiTe}_{3}$ and $\mathrm{TcGeSe}_{3}$ can be as large as $4 \times 10^{2}(\Omega \mathrm{cm})^{-1}$. These values are comparable to the intrinsic $\sigma_{x y}$ in some ferromagnetic metals, such as $7.5 \times 10^{2}(\Omega \mathrm{cm})^{-1}$ in bcc Fe [25,26] and
$4.8 \times 10^{2}(\Omega \mathrm{cm})^{-1}$ in fcc Ni [27], due to the Berry curvature of band structures.

A large magneto-optical Kerr effect is also possible in 2D ferromagnetic materials with large SOC [38]. We investigated the MOKE for $\mathrm{TcSiTe}_{3}, \mathrm{TcGeSe}_{3}$, and $\mathrm{TcGeTe}_{3}$ monolayers. The Kerr rotation angle is given by

$$
\theta_{\mathrm{Kerr}}(\omega)=-\operatorname{Re} \frac{\epsilon_{x y}}{\left(\epsilon_{x x}-1\right) \sqrt{\epsilon_{x x}}},
$$

where $\epsilon_{x x}$ and $\epsilon_{x y}$ are the diagonal and off-diagonal components of the dielectric tensor $\epsilon$ and $\omega$ is the photon energy,
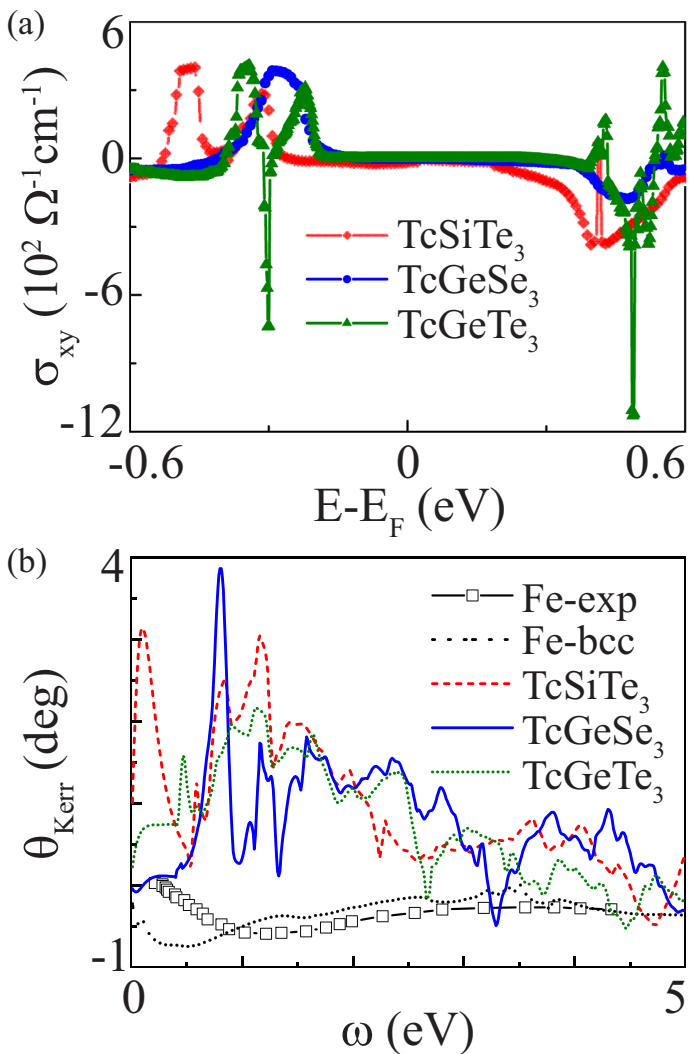

FIG. 5. (a) Anomalous Hall conductivity of $\mathrm{TcSiTe}_{3}, \mathrm{TcGeSe}_{3}$, and $\mathrm{TcGeTe}_{3}$ monolayers as a function of energy near the Fermi level. (b) Kerr angle $\theta_{\mathrm{Kerr}}$ of $\mathrm{TcSiTe}_{3}, \mathrm{TcGeSe}_{3}$, and $\mathrm{TcGeTe}_{3}$ monolayers as a function of photon energy, where the experimental (open squares) and calculated values (dotted line) of $\theta_{\text {Kerr }}$ for bulk Fe are included for comparison. 
TABLE II. Dominant hopping matrix elements $|V|$ and energy difference $\left|E_{p}-E_{d}\right|$ between $p$ orbitals of $\mathrm{Si}$ (Te) and $d$ orbitals of Tc (Cr) in $\mathrm{eV}$ for the $\mathrm{TcSiTe}_{3}\left(\mathrm{CrGeTe}_{3}\right)$ monolayer.

\begin{tabular}{lccccc}
\hline \hline Monolayer & Parameter & $p_{z}-d_{z^{2}}$ & $p_{z}-d_{x z}$ & $p_{z}-d_{y z}$ & $p_{z}-d_{x^{2}-y^{2}}$ \\
\hline $\mathrm{TcSiTe}_{3}$ & $|V|$ & 0.444869 & 0.096455 & 0.254298 & 0.179421 \\
$\mathrm{TcSiTe}_{3}$ & $\left|E_{p}-E_{d}\right|$ & 0.158048 & 0.118685 & 0.445139 & 0.296614 \\
Monolayer $_{\mathrm{CrGeTe}_{3}}$ & Parameter & $p_{x}-d_{z^{2}}$ & $p_{x}-d_{x z}$ & $p_{x}-d_{y z}$ & 0.386326 \\
$\mathrm{CrGeTe}_{3}$ & $|V|$ & 0.447729 & 0.216707 & 0.045947 & $p_{x}-d_{x^{2}-y^{2}}$ \\
\hline \hline
\end{tabular}

respectively. The dielectric tensor $\epsilon$ can be obtained by the optical conductivity tensor $\sigma(\omega)=\frac{\omega}{4 \pi i}[\epsilon(\omega)-I]$, where $I$ is the unit tensor. We performed the calculations with VASP along with the WANNIER90 tool to obtain the optical conductivity tensor $\sigma$ and the Kerr angle $\theta_{\text {Kerr }}$. The calculated $\theta_{\text {Kerr }}$ as a function of photon energy for $\mathrm{TcSiTe}_{3}, \mathrm{TcGeSe}_{3}$, and $\mathrm{TcGeTe}_{3}$ monolayers is shown in Fig. 5(b). Our calculated and previous experimental results for $\mathrm{Fe}$ metal are also included for comparison [39]. It can be seen that a large Kerr angle $\theta_{\text {Kerr }}$ is obtained for $\mathrm{TcSiTe}_{3}, \mathrm{TcGeSe}_{3}$, and $\mathrm{TcGeTe}_{3}$ monolayers, particularly for photon energies $\omega$ near $1 \mathrm{eV}$. The maximal Kerr angle for $\mathrm{TcSiTe}_{3}, \mathrm{TcGeSe}_{3}$, and $\mathrm{TcGeTe}_{3}$ monolayers is an order of magnitude larger than that for the $\mathrm{CrGeTe}_{3}$ monolayer [19] and about five times larger than that for bulk Fe.

\section{DISCUSSION}

How is the enhanced Curie temperature of the $\mathrm{TcSiTe}_{3}$ monolayer to be understood compared with that of the $\mathrm{CrGeTe}_{3}$ monolayer? According to the superexchange interaction [40-42], the FM coupling is expected since the Tc-Te$\mathrm{Tc}$ and $\mathrm{Cr}-\mathrm{Te}-\mathrm{Cr}$ bond angles are close to $90^{\circ}$. The indirect FM coupling between $\mathrm{Tc}(\mathrm{Cr})$ atoms is proportional to the direct AFM coupling between neighboring $\mathrm{Tc}(\mathrm{Cr})$ and Te atoms. The magnitude of this direct AFM coupling can be roughly estimated as $J=|V|^{2} /\left|E_{p}-E_{d}\right|$, where $|V|$ is the hopping matrix element between $p$ orbitals of Te and $d$ orbitals of Tc (Cr), and $\left|E_{p}-E_{d}\right|$ is the energy difference between $p$ orbitals of Te and $d$ orbitals of Tc (Cr). By using maximally localized Wannier orbital projections, the dominant hopping matrix elements $|V|$ and their corresponding energy differences $\left|E_{p}-E_{d}\right|$ can be obtained for $2 \mathrm{D} \mathrm{TcSiTe} 3$ and $\mathrm{CrGeTe}_{3}$, respectively, as listed in Table II. The results suggest that the direct AFM coupling for $\mathrm{TcSiTe}_{3}$ is dominated by the $p_{z}$ orbitals of Te and $d_{z^{2}}$ and $d_{x y}$ orbitals of Tc. Because the $p_{z}$ orbitals of Te and $d_{z^{2}}$ and $d_{x y}$ orbitals of Tc for the $\mathrm{TcSiTe}_{3}$ monolayer are very close to each other in energy and at the same time the sufficiently large hoppings exist between them, a large AFM coupling between Te and Tc atoms of $\mathrm{TcSiTe}_{3}$ is obtained. Although the hopping parameter is quite large between the $p_{x}$ orbitals of Te and $d_{z^{2}}$ and $d_{x^{2}-y^{2}}$ orbitals of $\mathrm{Cr}$ for the $\mathrm{CrGeTe}_{3}$ monolayer, because of the large energy differences among them, the AFM coupling between $\mathrm{Te}$ and $\mathrm{Cr}$ atoms is much weaker than that for the $\mathrm{TcSiTe}_{3}$ monolayer.

Are the giant orbital moments in $\mathrm{TcSiTe}_{3}, \mathrm{TcGeSe}_{3}$, and $\mathrm{TcGeTe}_{3}$ monolayers unique? To answer this question, we study the $2 \mathrm{D} M \mathrm{GeTe}_{3}$ monolayers with $M=3 d, 4 d, 5 d$ transition metals. The results of the orbital moment and magnetocrystalline anisotropy energy are listed in Fig. 6. From the spin-polarization calculations of these monolayers, only ten are found to be magnetic, and these magnetic materials are colored in red for $M$ metals in Fig. 6. Among these magnetic materials, the largest orbital moment is $0.53 \mu_{B}$ in $\mathrm{TcGeTe}_{3}$, which is two times larger than the second largest orbital moment in $\mathrm{CoGeTe}_{3}$ and about an order of magnitude larger than the orbital moment in the rest of the $2 \mathrm{D}$ materials. The same unique behavior of Tc is also found in the results of MAE. As listed in Fig. 6, one may find that among these 2D $\mathrm{MGeTe}_{3}$ materials, 2D $\mathrm{TcGeTe}_{3}$ has an extraordinarily large MAE.

\section{CONCLUSION}

Using first-principles calculations, we proposed three stable 2D Ising-type ferromagnetic semiconductors of $\mathrm{TcSiTe}_{3}$, $\mathrm{TcGeSe}_{3}$, and $\mathrm{TcGeTe}_{3}$ with high Curie temperatures of

\begin{tabular}{|c|c|c|c|c|c|c|c|c|c|}
\hline $\mathrm{Sc}$ & $\begin{array}{c}\text { Ti } \\
0.057 \\
1.0\end{array}$ & $\begin{array}{c}\mathbf{V} \\
0.078 \\
0.4\end{array}$ & $\begin{array}{c}\mathrm{Cr} \\
0.004 \\
0.93\end{array}$ & $\begin{array}{c}\text { Mn } \\
0.004 \\
1.3\end{array}$ & $\begin{array}{c}\mathbf{F e} \\
0.107 \\
0.3\end{array}$ & $\begin{array}{c}\text { Co } \\
0.233 \\
4.0\end{array}$ & $\begin{array}{c}\mathbf{N i} \\
0.090 \\
1.8\end{array}$ & $\mathrm{Cu}$ & $\mathrm{Zn}$ \\
\hline $\mathrm{Y}$ & $\mathrm{Zr}$ & $\mathrm{Nb}$ & $\begin{array}{c}\text { Mo } \\
0.023 \\
0.2\end{array}$ & $\begin{array}{c}\mathrm{Tc} \\
0.562 \\
28.0\end{array}$ & $\mathrm{Ru}$ & $\mathrm{Rh}$ & $\mathrm{Pd}$ & $\mathrm{Ag}$ & $\mathrm{Cd}$ \\
\hline & Hf & $\mathrm{Ta}$ & $\begin{array}{c}\mathbf{W} \\
0.021 \\
0.5\end{array}$ & $\mathrm{Re}$ & Os & Ir & $\mathrm{Pt}$ & $\mathrm{Au}$ & $\mathrm{Hg}$ \\
\hline
\end{tabular}

FIG. 6. Orbital moment (number in the first line, in units of $\mu_{B}$ ) and magnetocrystalline anisotropy energy (number in the second line, in units of $\mathrm{meV}$ ) for $M \mathrm{GeTe}_{3}\left(M=3 d, 4 d, 5 d\right.$ metals). That the compound $M \mathrm{GeTe}_{3}$ is a magnetic material is indicated by $M$ in red. The $\mathrm{MAE}$ is calculated by the energy difference per $M$ atom between the $\mathrm{FM}^{z}$ and $\mathrm{FM}^{x}$ configurations. 
538, 212, and $187 \mathrm{~K}$, respectively. Due to large spin-orbit couplings, the large magnetocrystalline anisotropy energy, large anomalous Hall conductivity, and large magneto-optical Kerr effect were found in these intriguing 2D ferromagnetic semiconductors. Comparing all possible $2 \mathrm{D} M \mathrm{GeTe}_{3}$ materials ( $M=3 d, 4 d, 5 d$ transition metals), the unique behavior of Tc was highlighted by an extraordinarily large orbital moment near $0.5 \mu_{B}$. The large orbital moments were revealed to be from the comparable crystal fields and electron correlations in these Tc-based 2D materials. The microscopic mechanism of the high Curie temperature was also addressed. Our findings present a series of materials with large spin-orbit coupling that could have essential implications in designing spintronic devices for next-generation microelectronics.

\section{ACKNOWLEDGMENTS}

G.S. was supported in part by the National Key R\&D Program of China (Grant No. 2018YFA0305800), the Strategic Priority Research Program of the Chinese Academy of Sciences (Grants No. XDB28000000 and No. XBD07010100), the National Natural Science Foundation of China (Grant No. 11834014), and Beijing Municipal Science and Technology Commission (Grant No. Z118100004218001). B.G. was supported by the National Natural Science Foundation of China (Grant No. Y81Z01A1A9), the Chinese Academy of Sciences (Grant No. Y929013EA2), and the University of Chinese Academy of Sciences (Grant No. 110200M208), and the Beijing Natural Science Foundation (Grant No. Z190011).
[1] A. Soumyanarayanan, N. Reyren, A. Fert, and C. Panagopoulos, Nature (London) 539, 509 (2016).

[2] M. T. Johnson, P. J. H. Bloemen, F. J. A. den Broeder, and J. J. de Vries, Rep. Prog. Phys. 59, 1409 (1996).

[3] M. Wu, J. Jiang, and M. Weng, Phys. Rep. 493, 61 (2010).

[4] D. L. Mills and S. M. Rezende, in Spin Dynamics in Confined Magnetic Structures II, edited by B. Hillebrands and K. Ounadjela, Topics in Applied Physics Vol. 87 (Springer, Berlin, 2003), pp. 27-59.

[5] T. R. McGuire, R. D. Hempstead, and S. Krongelb, in Magnetism and Magnetic Materials, edited by J. J. Becker, G. H. Lander, and J. J. Rhyne, AIP Conf. Proc. No. 29 (AIP, New York, 1976), p. 526.

[6] N. Nagaosa, J. Sinova, S. Onoda, A. H. MacDonald, and N. P. Ong, Rev. Mod. Phys. 82, 1539 (2010).

[7] A. Manchon, H. C. Koo, J. Nitta, S. M. Frolov, and R. A. Duine, Nat. Mater. 14, 871 (2015).

[8] A. Manchon and A. Belabbes, in Solid State Physics (Elsevier, Amsterdam, 2017), pp. 1-89.

[9] I. M. Miron, K. Garello, G. Gaudin, P.-J. Zermatten, M. V. Costache, S. Auffret, S. Bandiera, B. Rodmacq, A. Schuhl, and P. Gambardella, Nature (London) 476, 189 (2011).

[10] L. Liu, C.-F. Pai, Y. Li, H. W. Tseng, D. C. Ralph, and R. A. Buhrman, Science 336, 555 (2012).

[11] K. Garello, C. O. Avci, I. M. Miron, M. Baumgartner, A. Ghosh, S. Auffret, O. Boulle, G. Gaudin, and P. Gambardella, Appl. Phys. Lett. 105, 212402 (2014).

[12] I. M. Miron, T. Moore, H. Szambolics, L. D. BudaPrejbeanu, S. Auffret, B. Rodmacq, S. Pizzini, J. Vogel, M. Bonfim, A. Schuhl, and G. Gaudin, Nat. Mater. 10, 419 (2011).

[13] S.-H. Yang, K.-S. Ryu, and S. Parkin, Nat. Nanotechnol. 10, 221 (2015)

[14] W. Jiang, P. Upadhyaya, W. Zhang, G. Yu, M. B. Jungfleisch, F. Y. Fradin, J. E. Pearson, Y. Tserkovnyak, K. L. Wang, O. Heinonen, S. G. E. te Velthuis, and A. Hoffmann, Science 349, 283 (2015).

[15] S. Woo, K. Litzius, B. Krüger, M.-Y. Im, L. Caretta, K. Richter, M. Mann, A. Krone, R. M. Reeve, M. Weigand, P. Agrawal, I. Lemesh, M.-A. Mawass, P. Fischer, M. Kläui, and G. S. D. Beach, Nat. Mater. 15, 501 (2016).
[16] W. Jiang, X. Zhang, G. Yu, W. Zhang, X. Wang, M. B. Jungfleisch, J. E. Pearson, X. Cheng, O. Heinonen, K. L. Wang, Y. Zhou, A. Hoffmann, and S. G. E. te Velthuis, Nat. Phys. 13, 162 (2016).

[17] K. S. Burch, D. Mandrus, and J.-G. Park, Nature (London) 563, 47 (2018).

[18] B. Huang, G. Clark, E. Navarro-Moratalla, D. R. Klein, R. Cheng, K. L. Seyler, D. Zhong, E. Schmidgall, M. A. McGuire, D. H. Cobden, W. Yao, D. Xiao, P. Jarillo-Herrero, and X. Xu, Nature (London) 546, 270 (2017).

[19] C. Gong, L. Li, Z. Li, H. Ji, A. Stern, Y. Xia, T. Cao, W. Bao, C. Wang, Y. Wang, Z. Q. Qiu, R. J. Cava, S. G. Louie, J. Xia, and X. Zhang, Nature (London) 546, 265 (2017).

[20] X.-J. Dong, J.-Y. You, B. Gu, and G. Su, Phys. Rev. Appl. 12, 014020 (2019).

[21] N. D. Mermin and H. Wagner, Phys. Rev. Lett. 17, 1133 (1966).

[22] Y. Suzuki, T. Katayama, S. Yoshida, K. Tanaka, and K. Sato, Phys. Rev. Lett. 68, 3355 (1992).

[23] W. R. Bennett, W. Schwarzacher, and W. F. Egelhoff, Phys. Rev. Lett. 65, 3169 (1990).

[24] D. Weller, H. Brändle, and C. Chappert, J. Magn. Magn. Mater. 121, 461 (1993).

[25] Y. Yao, L. Kleinman, A. H. MacDonald, J. Sinova, T. Jungwirth, D. S. Wang, E. Wang, and Q. Niu, Phys. Rev. Lett. 92, 037204 (2004).

[26] X. Wang, J. R. Yates, I. Souza, and D. Vanderbilt, Phys. Rev. B 74, 195118 (2006).

[27] X. Wang, D. Vanderbilt, J. R. Yates, and I. Souza, Phys. Rev. B 76, 195109 (2007)

[28] G. Kresse and J. Furthmuller, Phys. Rev. B 54, 11169 (1996).

[29] P. E. Blochl, Phys. Rev. B 50, 17953 (1994).

[30] J. P. Perdew, K. Burke, and M. Ernzerhof, Phys. Rev. Lett. 77, 3865 (1996).

[31] J. Mravlje, M. Aichhorn, and A. Georges, Phys. Rev. Lett. 108, 197202 (2012).

[32] H. J. Monkhorst and J. D. Pack, Phys. Rev. B 13, 5188 (1976).

[33] A. Togo and I. Tanaka, Scr. Mater. 108, 1 (2015).

[34] A. A. Mostofi, J. R. Yates, G. Pizzi, Y.-S. Lee, I. Souza, D. Vanderbilt, and N. Marzari, Comput. Phys. Commun. 185, 2309 (2014).

[35] See Supplemental Material at http://link.aps.org/supplemental/ 10.1103/PhysRevResearch.2.013002 for details. 
[36] H. Xiang, C. Lee, H.-J. Koo, X. Gong, and M.-H. Whangbo, Dalton Trans. 42, 823 (2013).

[37] U. Wolff, Phys. Rev. Lett. 62, 361 (1989).

[38] B. Gu, S. Takahashi, and S. Maekawa, Phys. Rev. B 96, 214423 (2017).
[39] G. Krinchik and V. Artem'ev, Sov. Phys. JETP 26, 1080 (1968).

[40] J. B. Goodenough, Phys. Rev. 100, 564 (1955).

[41] J. Kanamori, J. Appl. Phys. 31, S14 (1960).

[42] P. W. Anderson, Phys. Rev. 115, 2 (1959). 